\title{
Linear Stapler in Total Laryngectomy
}

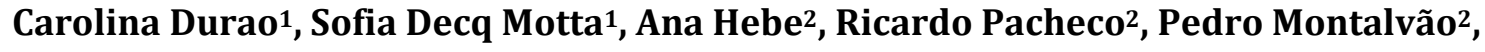 \\ Miguel Magalhães ${ }^{2}$ \\ ${ }^{1}$ Otolaryngology Department, Hospital Prof. Doutor Fernando Fonseca, Amadora, Portugal \\ ${ }^{2}$ Otolaryngology Department, Portuguese Oncology Institute of Lisbon, Francisco Gentil, Portugal \\ Email: carolinapinheirodurao@gmail.com
}

Received 21 September 2014; revised 20 October 2014; accepted 10 November 2014

Copyright (C) 2014 by authors and Scientific Research Publishing Inc.

This work is licensed under the Creative Commons Attribution International License (CC BY).

http://creativecommons.org/licenses/by/4.0/

C) (†) Open Access

\begin{abstract}
Introduction: Stapler application for pharyngeal closure after total laryngectomy allows rapid watertight closure. We intend to report the experience of the Portuguese Oncology Institute of Lisbon, Francisco Gentil (IPOLFG). Material and Methods: Retrospective study of patients submitted to total laryngectomy using linear stapler device treated in IPOLFG from 2005 to 2010. Results: 108 patients were studied. The majority of patients were male, aged from 60 to 69 years old, and had smoking and alcohol habits. The average length of hospital stay was 13.1 days. Post-operative complications occurred as follows: wound infection in $6.5 \%$, cervical hematoma in $4.6 \%$ and pharyngocutaneous fistula in $\mathbf{1 1 . 1 \%}$ of cases. Conclusions: The mechanical suture of the pharynx in total laryngectomy is a simple and quick method. It does not increase the incidence of post-operative complications. It seems to be a very safe method, as long as its limits regarding the location and extent of tumour are respected.
\end{abstract}

\section{Keywords}

Pharyngeal Closure, Total Laryngectomy, Linear Stapler

\section{Introduction}

Since the first total laryngectomy performed in 1873 by Theodor Billroth [1] there has been great progress. Nowadays some controversies still exist, namely, regarding the ideal method for closure of the pharyngeal defect created after total laryngectomy.

The requirements for pharyngoesophageal closure are absence of tension on wound edges and the possibility to preserve of viability of the mucosa, in order to create a waterproof barrier that contains pharyngeal secretions [2]. Closure of the pharynx is one of the most important surgical steps and requires special attention from the surgeon. The efficacy of this closure influences postoperative recovery and may be determinant in the occurrence of post-operative complications, particularly pharyngocutaneous fistula.

How to cite this paper: Durao, C., Motta, S.D., Hebe, A., Pacheco, R., Montalvão, P. and Magalhães, M. (2014) Linear Stapler in Total Laryngectomy. International Journal of Otolaryngology and Head \& Neck Surgery, 3, 354-358. 
Originally, hand suture closure of the pharynx was the only method available. But in recent decades, the closure of the pharynx with linear stapler during total laryngectomy has been popularized.

In the Portuguese Oncology Institute of Lisbon, Francisco Gentil (IPOLFG) the use of linear stapler for pharynx closure is performed with closed technique and exclusively on endolaryngeal tumours. We believe that this technique, if applied to such cases, allows a safe oncologic surgery. It should not be applied to larynx tumours where it is not possible to guarantee adequate tumour-free surgical margins [2]-[5].

When the resection of the larynx is performed as usual, it implies opening the pharynx and contaminating the surgical field with pharyngeal secretions. Alternatively, with linear stapler closed technique, the larynx is detached from the pharynx only when the closure of the pharynx is complete. This converts a surgery with a septic time into an aseptic intervention and might reduce postoperative complications [5].

Currently several authors advocate linear stapler closure numerous advantages comparing to the manual closure [2] [4]-[7].

It has been reported that hand suture closure is associated with an increase of operative time, and tissue necrosis [2] [6]. Necrosis is most likely induced by repeated manipulation of the mucosa by surgical instruments, or by the ischaemia induced by suture's wires. Conversely, the linear stapling machine allows a secure, haemostatic closure, with little tissue injury or inflammation [6].

Some even suggest that the linear stapler is associated with a lower rate of post-operative complications, namely pharyngocutaneous fistula, especially in previously irradiated patients or in those who undergo organ preservation protocols with chemotherapy associated with radiotherapy [4].

We also consider the fact that there is less variability among surgeons in pharyngeal closure with linear stapler than with manual closure [2]. Linear stapler allows that the pharynx closure depends more on local tissue factors, rather than on individual manual closure technique.

In order to clarify some of these aspects, the aim of this study is to report the experience of the Portuguese Oncology Institute of Lisbon, Francisco Gentil (IPOLFG) with the use of linear stapler for closure of the pharynx.

\section{Material and Methods}

We conducted a retrospective study of patients from IPOLFG, who, in the period from 2005 to 2012, underwent total laryngectomy for squamous cell carcinoma and closure of the pharynx with linear stapler. We included patients previously submitted to radiotherapy alone or combined with chemotherapy and patients with tracheotomy prior to surgery.

The population was characterized epidemiologically. Initial therapeutic approach and concomitant surgical procedures were characterized. Post-operative in-hospital stay, beginning of oral feeding and rate of surgical complications were evaluated.

The decision regarding the type of pharynx closure was made based on pre-operative endoscopy and computed tomography. In some cases it was complemented with suspension laryngoscopy under general anaesthesia and peri-operative exploration of the pharynx and tongue base. Mechanical suture was applied exclusively in endolaryngeal tumours with indication for total laryngectomy. Cases in which there was extralaryngeal tumour involvement, or when it was not possible to ensure adequate surgical margins were excluded.

Mechanical linear suture was applied with a stapling machine that enables application of two parallel rows of titanium staples. Firstly, the instrument approximates the edges of the tissue to suture. Then, when the trigger is activated, it fires staples. In theory, this double row of staples ensures the formation of an impermeable barrier between the neopharynx and the remaining cervical tissues. The model available at the IPOLFG is TX 60, which performs a linear suture of $60 \mathrm{~mm}$ long, with titanium staples.

IPOLFG uses linear stapler for pharynx closure with closed technique. Initially, the larynx is separated from its muscular and neurovascular attachments. After that, transection of the trachea is done, as well as the separation between the larynx and cervical oesophagus, keeping the pharynx closed. At this stage, in which resection of the larynx is imminent, the epiglottis is fixed in anterior position in order to avoid being included in the suture line. It is important to ensure that the entire tumour is completely contained in the piece to remove and that the surgical margins are adequate. After this, the linear stapling machine is applied longitudinally between the pharynx and the larynx and the closest to the thyroid cartilage, in order to preserve as much as possible of healthy pharyngeal mucosa (Figure 1). When all these steps are completed, the trigger of the linear stapler may be securely activated, thus, creating a double suture line. At this stage, the pharynx is separated from the larynx without contaminating the operating field with pharyngeal secretions (Figure 2). After this, the linear stapling 


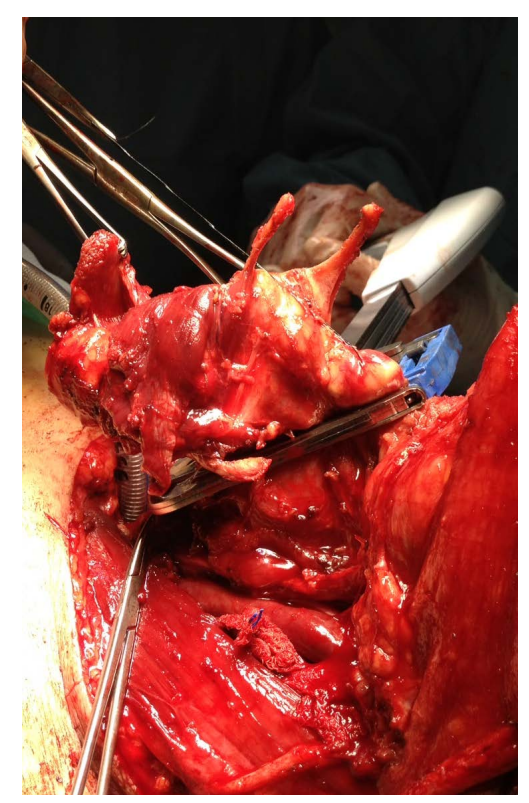

Figure 1. Linear stapler placed between larynx and the pharynx.

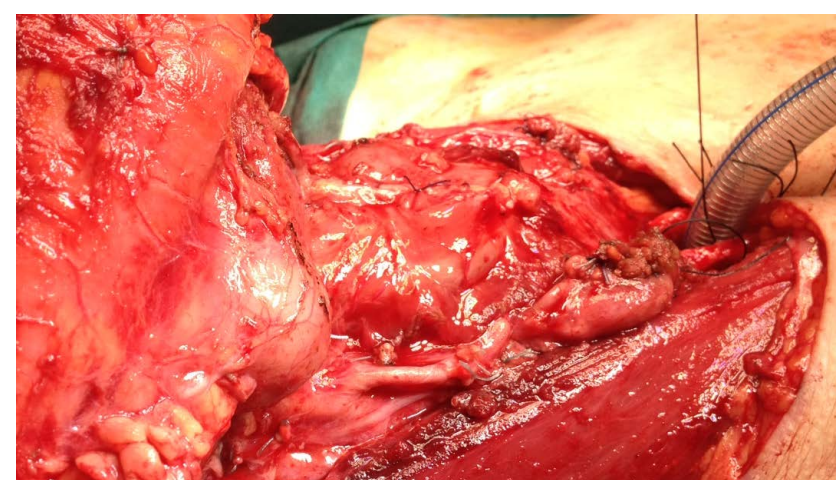

Figure 2. Mechanical suture of the pharynx.

machine is removed and the suture is inspected in order to confirm that it is securely watertight. The following steps are similar to those used in the conventional technique.

\section{Results}

A total of 474 total laryngectomies were performed from 2005 to 2010. Of these, 108 patients (22.6\%) underwent closure of the pharynx with linear stapler. In the 108 patients, the predominant age group was 60 - 69 years old. There was male predominance (97.2\%) and the majority had smoking habits (74\%). Fifty (50\%) had moderate to severe drinking habits. The most frequent presenting symptoms of laryngeal cancer were dyspnea and dysphonia. In $28.7 \%$ of cases, dyspnea led to emergency tracheotomy before surgery.

In our population, the initial treatment plan was surgery in 103 patients (95.4\%). The remaining 4.6\% were proposed to organ preservation protocol and, since they have not responded, salvage surgical treatment was performed. Of the 103 patients to whom surgery was proposed as initial treatment, 5 patients had radiotherapy and 1 patient had prior chemotherapy and radiotherapy for other tumours of the head and neck. In summary, surgery was carried out in $89.8 \%$ of cases without prior irradiation and with prior radiotherapy in $10.1 \%$ of cases.

Total laryngectomy with linear stapler was associated with bilateral neck dissection in 104 patients (96.3\%). Voice prosthesis was applied in 10 patients (9.3\%). Sporadically and whenever it was considered oncologically relevant, the emptying of the recurrential chain and/or a hemithyroidectomy was also performed. In one case, a myocutaneous flap of large pectoral was made. 
Regarding the post-operative period, the average hospital stay was 13.1 days. Oral intake was started on average at the 12th post-operative day.

Five patients (4.6\%) had post-operative cervical haematoma, twelve patients $(11.1 \%)$ had salivary fistula and surgical wound infection occurred in 7 patients (6.5\%). In one case there was a lymph fistula that motivated a revision surgery and ligation of the thoracic duct.

The vast majority of the salivary fistulae closed spontaneously (83.3\%). The time for spontaneous closure ranged from 22 to 406 days. Of these 12 patients, only one patient had undergone radiotherapy prior to surgery.

Mean post-operative follow-up was 36.7 months (minimum 0.5 and maximum 87.9). In our series, $77.8 \%$ of patients were proposed for adjuvant radiotherapy, 5.5\% received adjuvant chemoradiotherapy, and the remaining $16.7 \%$ did not carry out any adjuvant treatment. Most (90\%) patients with voice prosthesis successfully performed speech rehabilitation. Sixteen patients reported dysphagia several months after surgery and radiotherapy. There was oesophageal stenosis requiring oesophageal dilatation in $87.5 \%$ of these cases. In 3 cases there was salivary fistula after surgery and radiotherapy.

Overall survival at 2 years was $80.9 \%$ and at 5 years was $50.4 \%$.

\section{Discussion}

Demographic characteristics of our population are similar with the ones from the literature, confirming the predominance of cancer of the larynx in men between 50 and 70 years old with smoking and drinking habits. In $28.7 \%$ of the cases, dyspnea led to emergency tracheotomy before surgery. The surgery was performed in $89.8 \%$ of cases without prior irradiation.

The linear stapler technique employed at the Portuguese Oncology Institute of Lisbon, Francisco Gentil (IPOLFG) is very similar to the technique used in other centres. There are, however, some differences that will be listed. According to Bedrin et al. [2], the manual suture of pre-laryngeal muscles is unnecessary to reinforce the suture of the pharynx; the IPOLFG performs it routinely. Sofferman et al. [5] apply linear stapler with 2 shots from linear stapling machine, creating a double row of staples; in IPOLFG the suture is performed with only one shot. Altissimi et al. [7] perform traction of the epiglottis using endoscopy and a variant of the closed technique to ensure that the epiglottis is not trapped in the suture line, but in IPOLFG the retraction is made by palpation without direct visualization. Agrawal et al. [8] use a stapling machine of $90 \mathrm{~mm}$ long, on the other hand, in the IPOLFG we use one of $60 \mathrm{~mm}$ long. Despite these small differences in technique the percentage of surgical complications was similar to that of other centers.

Regarding the duration of surgery, all authors are unanimous in noting that the use of linear stapler appears to decrease the time of the intervention. Montoya et al. [6] sought to objectify this idea and compared the surgical time of total laryngectomy by manual suture with that of TL by linear stapler. It states a difference of 43 minutes between each technique. This may be beneficial for patients with high anaesthetic risk and may also reduce the costs.

The in-hospital stay and the start of oral feeding are conditioned by the existence of post-operative complications. In our series, the average hospital stay was 13.1 days and the average start of oral feeding was on the 12th post-operative day. Montoya et al. [6] state an average period of hospital stay of 13.5 days and Ortega of 12.4 days.

The occurrence of complications, especially of pharyngocutaneous fistula, depends on several factors, including the suture method used in closure of the pharynx. In our series, there was wound infection in $6.5 \%$ of cases, cervical haematoma in $4.6 \%$ of cases and pharyngocutaneous fistula in $11.1 \%$ of cases. It should be noted that voice prosthesis were not applied in any of these, and that only one patient had undergone radiotherapy prior to surgery. The percentage of patients with pharyngocutaneous fistula was similar to other centres. Ahumada et al. [9] studied 36 patients of whom 8.7\% developed pharyngocutaneous fistula and Altissimi et al. [7] report a rate of $4.2 \%$ in a total of 70 patients with closure of the pharynx by linear stapler.

There are studies comparing the occurrence of pharyngocutaneous fistula between patients with closure of the pharynx by linear stapler and patients with closure of the pharynx by manual suture. They all report a lower percentage in the group subject to linear stapler with closed technique. Gonçalves et al. [4] report the occurrence of fistula in $36.7 \%$ of cases of manual suture and in $6.7 \%$ of cases of linear stapler. Montoya et al. [6] describe $27 \%$ of cases with pharyngocutaneous fistula in the group of manual sutures and $5 \%$ of cases in the group of linear stapler. In the future, it would be interesting to perform a similar comparison with patients of IPOLFG to 
ascertain whether there is a reduction of complications.

Finally, it is noted that the majority (90\%) of patients in which voice prosthesis was placed conducted speech rehabilitation successfully and that the cases of dysphagia in our series occurred only after radiotherapy. It seems that this method of closure of the pharynx does not compromise the use of the voice prosthesis. Cases of dysphagia cannot be attributed exclusively to the method of linear stapler.

The advantages and details of the use of linear stapling machines for performing the closure of the pharynx have been listed. The biggest disadvantage of linear stapler by closed technique is not allowing visualization of the tumour during resection. Linear stapler was conducted only in cases where it was possible to ensure adequate surgical margins and complete tumour resection. The average 5-year survival is similar to other studies, for one can admit that the application of this type of suture in the pharynx did not adversely affect the prognosis of the patients. It seems that if we confine its application to endolaryngeal tumours this technique is safe and allows an adequate oncologic surgery [6].

\section{Conclusion}

The mechanical suture of the pharynx in total laryngectomy is a simple and fast method with low risk of contamination of the surgical site. It does not increase the incidence of post-operative complications. It seems to be a safe method provided that the limits of its indication, as to the location and tumour extension are respected.

\section{Acknowledgements}

The authors thank all the other members of the Otolaryngology Department of Portuguese Oncology Institute of Lisbon, Francisco Gentil (IPOLFG) for scientific assistance.

\section{References}

[1] Stell, P.M. (1975) The First Laryngectomy. The Journal of Laryngology and Otology, 89, 353-358. http://dx.doi.org/10.1017/S0022215100080488

[2] Bedrin, L., Ginsburg, G., Horowitz, Z. and Talmi, Y.P. (2005) 25-Year Experience of Using a Linear Stapler in Laryngectomy. Head \& Neck, 27, 1073-1079. http://dx.doi.org/10.1007/s00405-009-0945-4

[3] Estibeiro, H. (2004) Total Laryngectomy, Surgery of the Larynx. N.P. Circulo Médico, 123-135.

[4] Gonçalves, A.J., Souza Jr., J.A., Menezes, M.B., Kavabata, N.K., et al. (2009) Pharyngocutaneous Fistulae Following Total Laryngectomy Comparison between Manual and Mechanical Sutures. European Archives of Oto-Rhino-Laryngology, 266, 1793-1798. http://dx.doi.org/10.1007/s00405-009-0945-4

[5] Sofferman, R.A. and Voronetsky, I. (2000) Use of the Linear Stapler for Pharyngoesophageal Closure after Total Laryngectomy. The Laryngoscope, 110, 1406-1409. http://dx.doi.org/10.1097/00005537-200008000-00035

[6] Montoya, F., Ruiz de Galarreta, J.C., Sánchez del Rey, A., Martínez Ibargüen, A., et al. (2002) Comparative Study between the Use of Manual versus Mechanical Sutures in the Closing of the Mucous Defect Following a Total Laryngectomy. Acta Otorrinolaringológica Española, 53, 343-350.

[7] Altissimi, G. and Frenguelli, A. (2007) Linear Stapler Closure of the Pharynx during Total Laryngectomy: A 15-Year Experience (from Closed Technique to Semi-Closed Technique). Acta Otorhinolaryngologica Itálica, 27, 118-122.

[8] Agrawal, A. and Schuller, E. (2000) Closed Laryngectomy Using the Automatic Linear Stapling Device. The Laryngoscope, 110, 1402-1405. http://dx.doi.org/10.1097/00005537-200008000-00034

[9] Ahumada, N., Oliveira, C. and Takimoto, R. (2011) Stapler Device at the Closure of the Pharynx after Total Laryngectomy: 7 Years of Experience. Revista Brasileira de Cirurgia da Cabeça e Pescoço, 40, 144-147. 
Scientific Research Publishing (SCIRP) is one of the largest Open Access journal publishers. It is currently publishing more than 200 open access, online, peer-reviewed journals covering a wide range of academic disciplines. SCIRP serves the worldwide academic communities and contributes to the progress and application of science with its publication.

Other selected journals from SCIRP are listed as below. Submit your manuscript to us via either submit@scirp.org or Online Submission Portal.
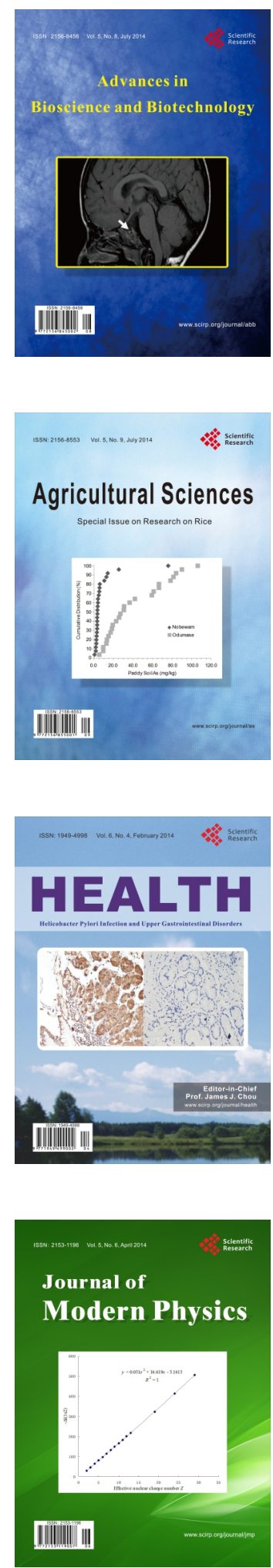
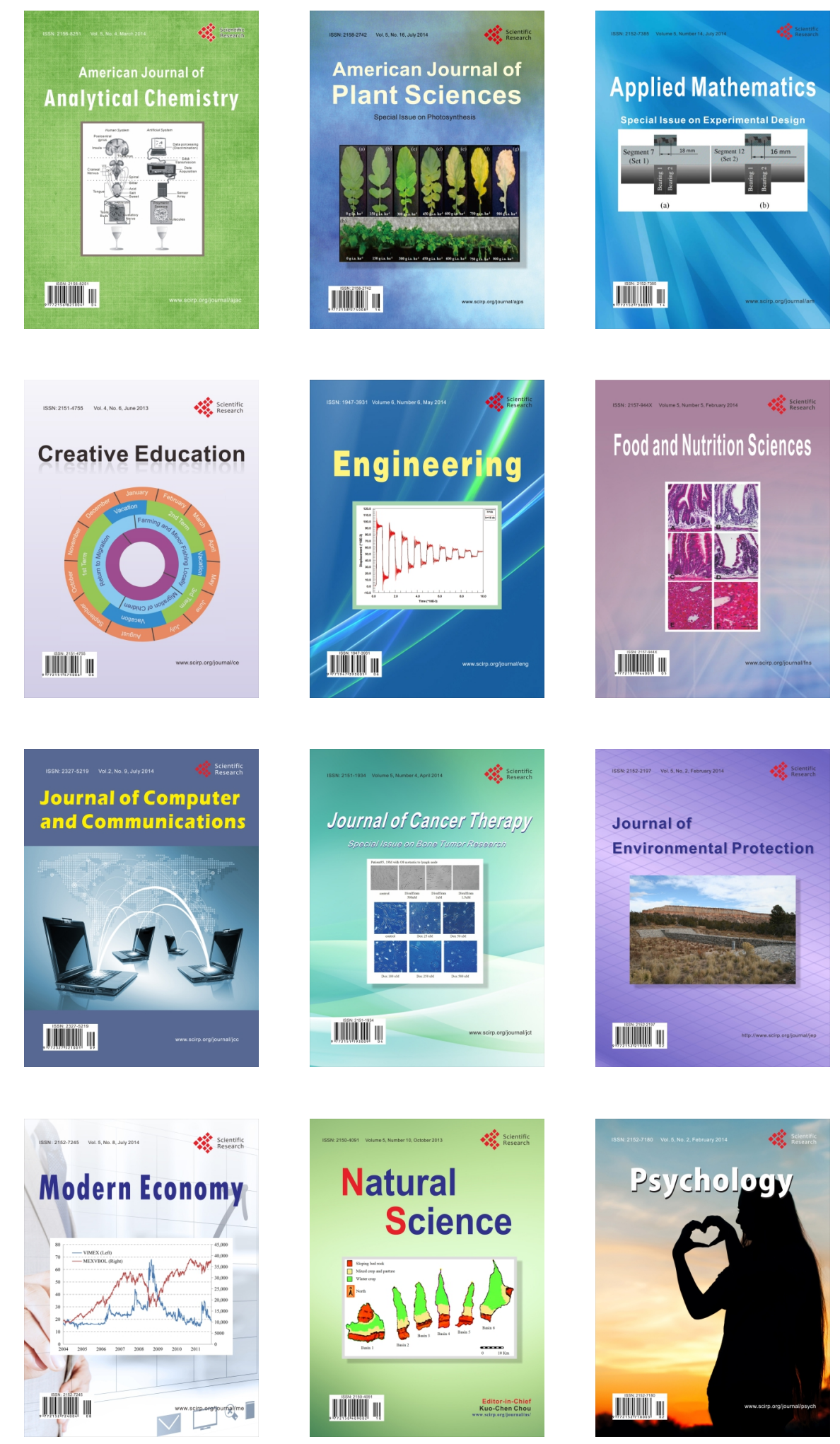